\title{
Surface vibration analysis (SVA): a new non-invasive monitor of gastrointestinal activity
}

\author{
F C CAMPBELl, B E STOREY, P T CULlEN, AND A CUSCHIERI \\ From the Depts of Surgery and Physics, Ninewells Hospital and Medical School, Dundee
}

SUMmary A computerised system for measurement of vibration at the abdominal surface was constructed which was addressed to the evaluation of gastrointestinal (GI) motor function. Preliminary studies revealed a dominant low frequency signal which was synchronous with the heartbeat and was considered representative of aortic pulsation. This was excluded by selective spectral filtration. The remaining signal was processed and measured by computer, with provision of quantitative energy values as well as of graphic display. The developed method, called surface vibration analysis (SVA) has been evaluated clinically; (a) against oral to caecal transit times (OCCT) of a standard solid meal, in five patients with severe postgastrectomy diarrhoea, seven patients with mild idiopathic diarrhoea and 22 healthy volunteers. (b) against prokinetic effects of a gastrointestinal stimulant (cisapride) in nine patients. In (a) postprandial SVA energy measurements were greater $\left.\left(\mathrm{SVA}{ }^{*} \overline{\mathrm{X}}(\mathrm{SEM})\right]=406933(98224)\right)$ and oral to caecal transit of the solid meal was more rapid (OCTT $=* 90(29) \mathrm{min})$ in the severe diarrhoea patients [postgastrectomy] than either the mild diarrhoea group $(* \mathrm{SVA}=235317(50780) ; *$ OCTT $=* 199(42)$ min $)$ or normal volunteers ( $*$ SVA $=212062(27153)$; $*$ OCTT $=242(19) \mathrm{min})$ [p $<0.01$ for SVA and OCTT]. In the total group, an inverse correlation was observed between quantitative SVA energy values and oral to caecal transit times of solids (Spearman's rho $=-0 \cdot 486 ; p<0 \cdot 01)$. In (b), drug stimulation of the GI tract caused an increase of fasting SVA measurements from *21 $217(5956)$ [before] to *41 $937(9606)$ [after] intravenous cisapride $(p<0 \cdot 05)$. This new technique may be useful for evaluation of gastrointestinal motor activity.

\begin{abstract}
A body in fluctuating motion releases vibrations to its surrounding medium. ' Cannon first drew attention to the concept that contractions of the alimentary tract, which are associated with movement of intraluminal contents and of the gut wall, yield vibrational energy to surrounding tissue and suggested that this energy could be captured at the abdominal wall for diagnostic purposes.' Subsequent work in this area has been scant and has involved the use of surface microphones, for signal capture. Susceptibility to background noise, however, was a major limitation of this methodology and furthermore, the short duration signals obtained made interpretation

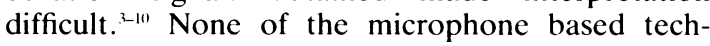

Address for correspondence: Mr F C Campbell, Dept of Surgery, Ninewells Hospital and Medical School, Dundec DD1 9SY.

Received for publication 21 July 1988.

*Patent pending.
\end{abstract}

niques were evaluated against any standard test of gastrointestinal activity.

This study describes a new method of capture and analysis of vibrational energy at the abdominal surface which provides quantitative as well as qualitative data. The method has been tested against standard measurements of gastrointestinal transit and its sensitivity for measurement of prokinetic gastrointestinal stimulation has also been investigated.

\section{Methods}

SURFACE VIBRATION ANALYSIS (SVA)

The SVA system* has been developed by collaboration between the Departments of Surgery and Physics, University of Dundee, over the past three years. The system was based upon a piezoelectric 
transducer which was designed for industrial vibration measurement (Bruel and Kjaer Instruments Ltd, Copenhagen, Denmark). This transducer has minimal response to acoustic background but converts vibrational energy transmitted directly through the abdominal wall into an electronic signal. The wide spectral frequency response of the transducer has been modified by incorporation of a high pass filter which provides - 60 decibel attenuation at 40 $\mathrm{Hz}$, to give a flat response between $40-10000 \mathrm{~Hz}$. After filtering, the signal was calibrated against a stable oscillator output, processed by precision (half wave) rectifier and amplified at fixed gain. The signal was then integrated over five second intervals using a gated analogue integrator with a sample and hold circuit. The output from this device was fed to the inbuilt analogue to digital converter, of a BBC microcomputer (Acorn Computers Ltd, Cambridge, England). Purpose designed software was used to integrate the signal further over intervals of one minute, with provision for the output of sequential and accumulated signal amplitude measurements and graphic display of the signal as a function of time.

A permanent hard copy record of the numerical and graphic results was produced on a linked dot matrix printer. Surface vibration analysis signals were also simultaneously stored on magnetic tape to permit subsequent frequency analysis, by Fast Fourier Transform (FFT)."

The transducer is supplied by the manufacturer, with a calibration figure which can be dialled to the pre-amplifier, thus providing for normalisation of all units and easy calibration. The scale of signal measurement by the SVA system, was defined empirically to achieve satisfactory deflection in the fasting state, in all normal subjects. This scale could be reproduced under laboratory conditions using a Bruel and Kjaer type 2034 vibrating table, with an acceleration setting between $0-40 \mathrm{~m} /$ second. $^{2}$ At these settings, a flat frequency response was obtained between $40 \mathrm{~Hz}$ and the table's upper frequency limit, of $6400 \mathrm{~Hz}$.

Quantitative SVA values were expressed in arbitrary linear energy units, which were constant in all studies.

\section{CLINICALEVALUATION}

SOLID MEAL STUDY

\section{Subjects}

This study was carried out in 22 healthy volunteers, seven patients with episodic diarrhoea of unknown cause and five patients who had undergone total gastrectomy. The ages of the three groups differed (Table). All volunteers were asymptomatic and none had undergone previous gastrointestinal surgery. The diarrhoea group had episodic trouble with up to five motions per day during crises whereas the postgastrectomy patients had persistent loose stools, with five to 10 motions per day on a regular basis.

\section{Study protocol}

All subjects had SVA recordings taken for 30 minutes in the fasting phase, followed by an eight minute pause for ingestion of their standard solid meal, during which the recording was stopped. After the meal, SVA recording was continued for three and a half hours. The oral to caecal transit time of the solid meal was measured simultaneously, by the hydrogen breath test (vide infra).

\section{DRUG STIMULATION STUDY}

\section{Subjects}

This study was carried out in nine patients with troublesome symptoms of 'gas bloat' syndrome, after antireflux surgery, which had been carried out at a mean of 4.8 years previously. These patients were selected for treatment with a new gastrointestinal stimulant, cisapride. This agent is a new synthetic, peripherally acting cholinergic agonist (Janssen Pharmaceutica, Grove, Wantage, Oxon, England). None of the patients in this group had diarrhoea.

\section{Study protocol}

In this study, baseline SVA recordings and measurements of oral to caecal transit time (OCTT) were taken in response to the standard solid meal in an identical manner to that outlined above.

Each patient acted as his own control and returned one week after baseline studies, for a repetition of the SVA and OCTT tests in response to intravenous cisapride, to obtain stimulated measurements. Cisapride was given as a single $(4 \mathrm{mg})$ intravenous bolus midway (15 minutes) through the fasting phase.

Table Subject measurements in solid meal study

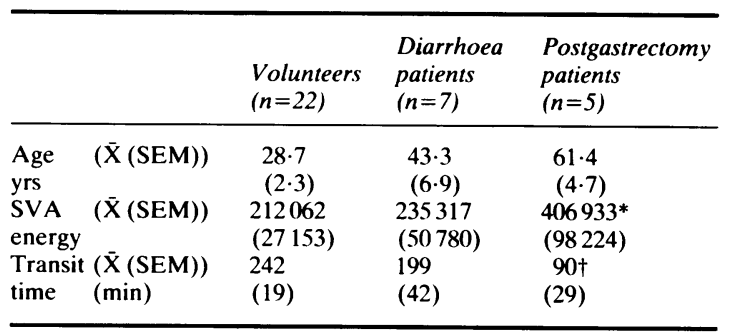

${ }^{*} \mathrm{p}<0.05+\mathrm{p}<0.01$ postgastrectomy patients $v$ normal subjects by Mann Whitney. No significant differences of transit time or of SVA energy values were observed between normals and episodic diarrhoea patients. 
As before, SVA recordings were stopped after 30 minutes fasting, for eight minutes, to allow meal ingestion and then continued for three and a half hours thereafter. Thus, in the stimulated test, SVA recordings were obtained of: (a) baseline activity for 15 minutes in the fasting phase, (b) stimulated activity for 15 minutes fasting and (c) stimulated activity for three and a half hours in the postprandial phase. Surface vibration analysis energy values were then compared with baseline studies in each patient. As before, oral to caecal transit times were evaluated simultaneously by the $\mathrm{H}_{2}$ breath test .

\section{SVA RECORDING}

In all clinical studies for this project, the transducer was positioned in the right upper quadrant of the abdomen, beneath the costal margin. Patients were required to lie still during recordings. Recording was continuous with interruptions only for ingestion of the meal (eight minutes) and for breath hydrogen sampling (approx 30 seconds ever 15 minutes).

\section{MEASUREMENT OF ORAL TO CAECAL TRANSIT TIME}

Intestinal transit in both parts of the study was monitored by the hydrogen breath technique..$^{12}$ In all cases, tests were carried out after a 12 hour fast, and subjects had been instructed to avoid foods containing non-absorbable saccharides such as bran, peas, beans, etc, for 24 hours, and to refrain from smoking for six hours, before the test. A chlorhexidine mouthwash was given to all subjects before the test, to eradicate oral bacteria. Hydrogen concentration was assessed using a GMI exhaled hydrogen monitor (GMI Instruments Ltd, Renfrew, Scotland), in $20 \mathrm{ml}$ samples of end alveolar air, in the fasting phase and at 15 minute intervals after the test meal, until the critical rise of 10 parts per million of breath $\mathrm{H}_{2}$ was observed, which was considered indicative of completion of oral to caecal transit time..$^{13}$

STANDARD SOLID MEAL

The standard solid meal in all studies comprised $200 \mathrm{~g}$ mashed potato, $100 \mathrm{~g}$ baked beans (substrate for hydrogen breath test), and $50 \mathrm{ml}$ water, which had a calorific value of $253 \mathrm{kcal}$.

DATA ANALYSIS

All frequency analysis and related signal processing was carried out using Interactive Laboratory System (ILS) software (Signal Technology Inc, Goletta, California, USA) running on an $\mathrm{IBM}^{\circledR}$ compatible microcomputer fitted with a 12 bit analogue to digital conversion card (Type AD2801A) (Data Translation Ltd, Marlborough, Massachussetts, USA).

The SVA signal varied as a function of time. Thus, for comparison of quantitative SVA data, total accumulated values for specific time intervals were addressed - for example, in the fasting phase, total SVA values for the 15 minute intervals before and after cisapride were compared. In the postprandial phase, total SVA values for the 210 minute interval after the solid meal, were evaluated against the oral to caecal transit time. In addition, the 210 minute total postprandial SVA values before and after cisapride, were compared.

STATISTICAL ANALYSIS

For normally distributed data, Student's $t$ test of significance was utilised for evaluation of differences between two unrelated samples. Wilcoxon's signedrank test was utilised for related samples, and the Mann Whitney $U$ test was used for unrelated samples of non-parametric data. Spearman's test $(\varrho=r h o)$ of correlation was used for evaluation of probability of correlation for non-parametric data.

\section{Results}

PRELIMINARY VIBRATION ANALYSIS

A repetitive high energy signal initially predominated. This was found by (FFT) frequency analysis, to occupy a low spectral frequency range, below 30 $\mathrm{Hz}$ (Fig. 1). Detailed examination of this signal by analysis of amplitude as a function of time, showed the regularity and rapid rate of rise and decay, consistent with a pulse (Fig. 2a). The repetition incidence of this signal was always synchronous in

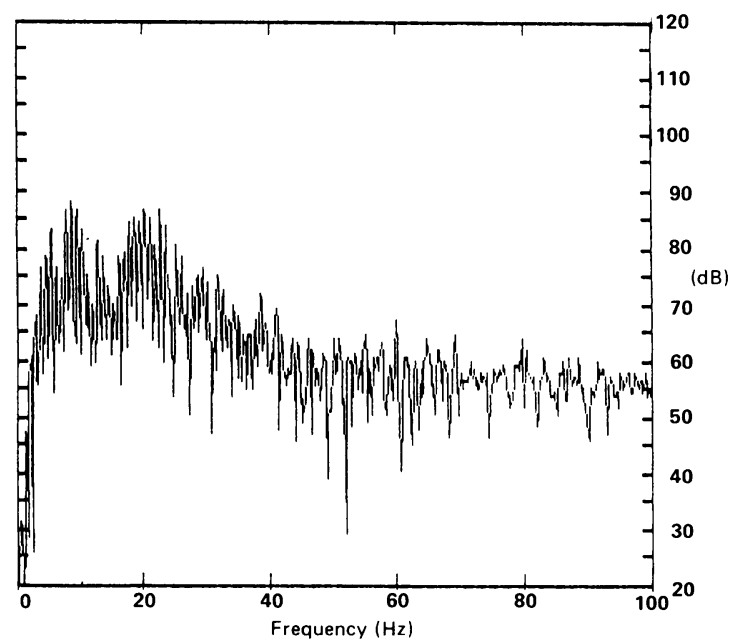

Fig. 1 Spectral frequency range of the dominant signal: Fast fourier transform analysis showed that the initial dominant signal peaked at a spectral frequency range of between $0-30$ $\mathrm{Hz}$ at $+25-30$ decibels above baseline. 

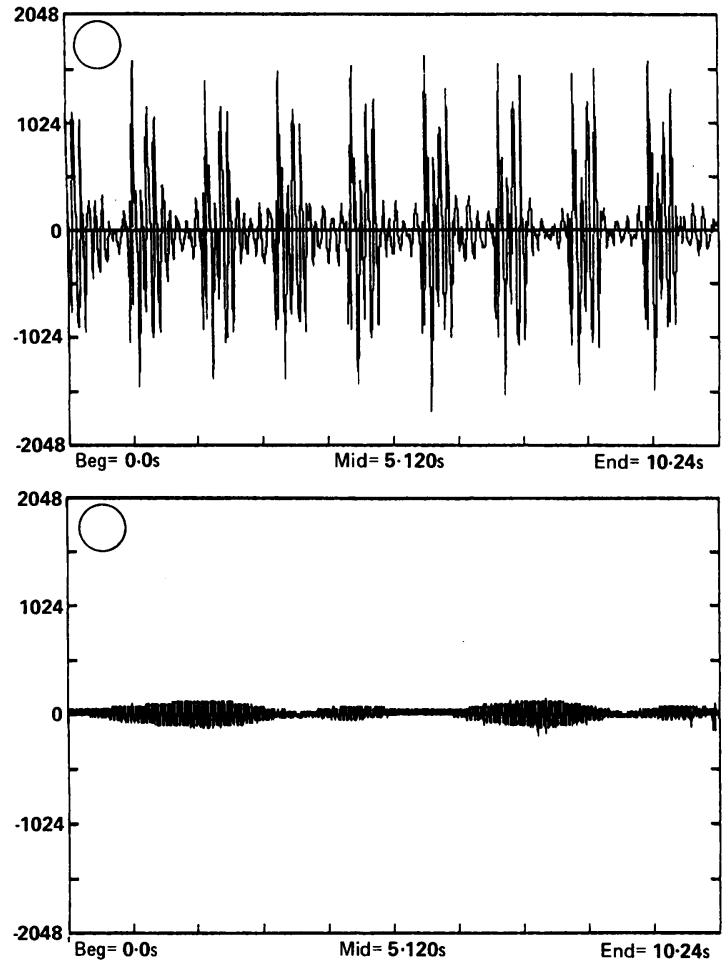

Fig. 2 (a) Analysis of signal amplitude as a function of time: The signal shown has been captured over a 10.24 second interval, and has been neither processed nor filtered by the $S V A$ system. Note the repetitive high energy tracing, with rapid rise and decay consistent with an energy pulse. The repetition rate of 54/minute was exactly synchronous with the heartbeat. This dominant signal was considered to be representative of aortic pulsation. (b) Signal attenuation by high pass filter. This is the same signal as shown in Fig. $2 a$, but it has been processed by the SVA system, utilising a 4 pole high pass filter. Note the considerable attenuation of the dominant pulsatile signal, which has been achieved.

time with the directly monitored heartbeat. Attenuation was achieved by the incorporation of a four pole high pass filter which eradicated the pulsatile signal with minimum effect on the SVA signal at higher spectral frequencies (Fig. 2b). The final SVA system incorporated this signal conditioning circuitry.

\section{CLINICAL EVALUATION OF THE DEVELOPED} SYSTEM

\section{Solid meal study}

The characteristics of the subjects in the three groups differed (Table). Transit times of solids ranged from 30 to 400 minutes $(\bar{X}(\mathrm{SEM})=211 \cdot 3(15 \cdot 7) \mathrm{min})$ and postprandial SVA values ranged from 84518 to 790577 with a sample median of 200470 . Transit

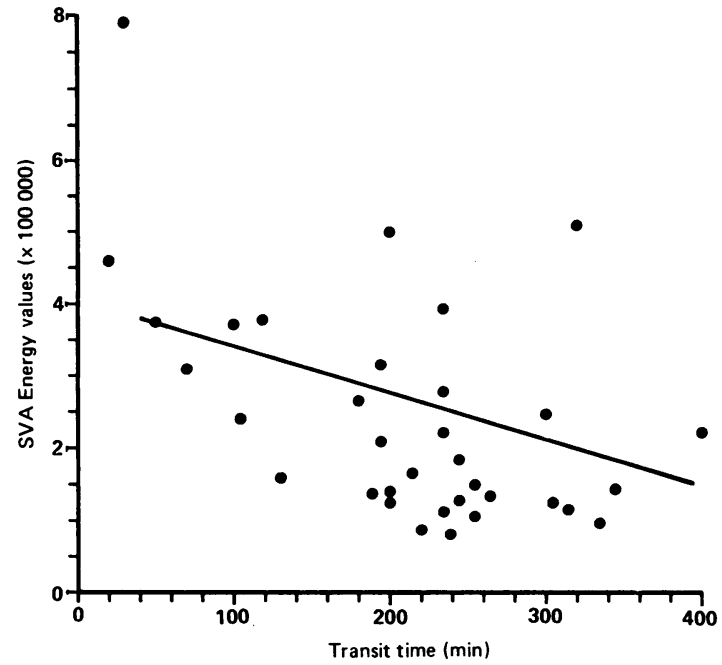

Fig. 3 Correlation between quantitative postprandial SVA energy values and oral to caecal transit time of solids: The measurements were taken over intervals of three and $a$ half hours, in 22 volunteers, seven patients with episodic diarrhoea and five patients after a total gastrectomy. Spearman's rho $(\mathrm{Q})=-0.486 ; 32 d f ; p<0 \cdot 01$.

times were shorter $(\mathrm{p}<0.01)$ and postprandial SVA vaues were greater $(p<0.05)$ in post gastrectomy patients, when compared to volunteers (Table). An inverse correlation was observed in the total group, between oral to caecal transit times and postprandial SVA energy values $(\varrho=-0.486 ; p<0.01)$ (Fig. 3$)$. The graphic display of the SVA system showed a

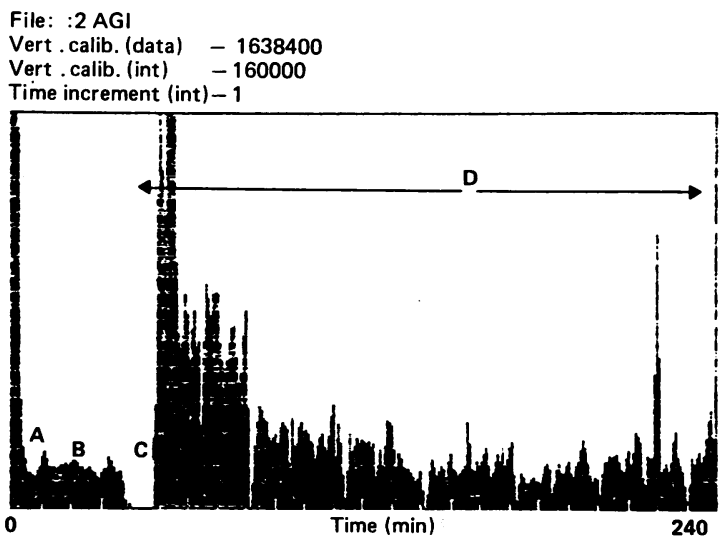

Fig. 4 Response to a standard solid meal as shown by SVA graphic display: The components of this baseline - that is, before cisapride tracing are as shown: $A$-Calibration signal; $B$ - Fasting activity; $C$-Pause for the solid meal (eight minutes) (note that the whole SVA system is switched off during this time); D-Postprandial response to the meal. Note the high early peak lasting approximately 60 minutes with a more gradual return to baseline thereafter. 


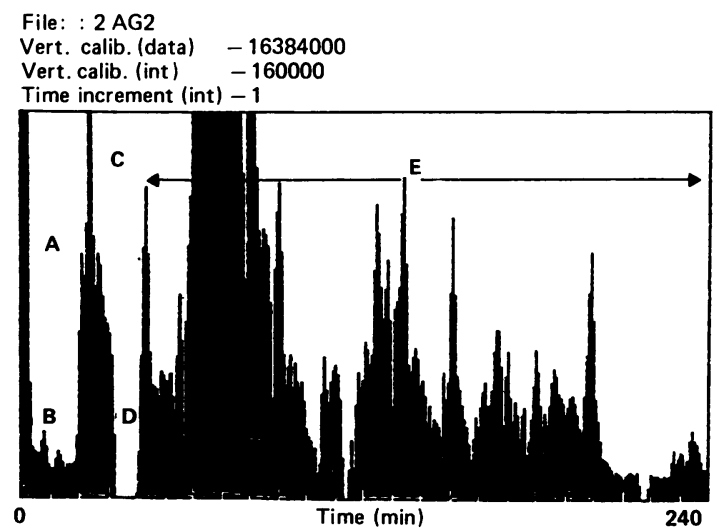

Fig. 5 Response to a standard solid meal after cisapride stimulation: The components of this stimulated tracing are as shown: A - Calibration signal; $B$-Unstimulated fasting activity; $C$-Stimulated fasting activity peak; D-Pause for solid meal (eight minutes); $E$-Stimulated postprandial activity. Note that this tracing was taken from the same individual (subject AG), after the same standard meal as in Fig. 5, but this time with cisapride stimulation.

typical high early postprandial energy peak after the solid meal, which generally subsided within 20-60 minutes (Fig. 4).

\section{Drug stimulation study}

The ages of the patients range from 34-76 years $(\bar{X}(\mathrm{SEM})=60.9(6 \cdot 2)$ years $)$. Intravenous cisapride promoted intestinal transit, with a reduction of oral to caecal transit times from $(\overline{\mathrm{X}}(\mathrm{SEM})=132 \cdot 78(17 \cdot 5)$ $\min )$ before to $(\overline{\mathrm{X}}(\mathrm{SEM})=70.0(7.9) \mathrm{min})$ after cisapride $[\mathrm{p}<0.01$ by Mann Whitney]. The graphic display of the SVA system clearly showed the effect of Cisapride stimulation of GI activity (Fig. 5) when compared with baseline (Fig. 4). Fasting quantitative SVA energy values were significantly greater after cisapride stimulation $(\bar{X}(S E M)=41937(9606))$ than in the baseline state $(\bar{X}(\mathrm{SEM})=21217$ (5956)) [p<0.05] (Fig. 6), although differences in fed values failed to reach statistical significance.

\section{Discussion}

Previous work in this area, has attempted to capture acoustic energy emanating from gastrointestinal contractions over short intervals, by the use of surface microphones. ${ }^{3-10}$ The present system departs from this concept and relies on a surface piezeolectric transducer which by direct coupling, measures sinusoidal displacement of the vibrating abdominal surface and avoids the inefficiency of the additional interface of air, which is necessary for microphones. Thus, the present method has the advantages of

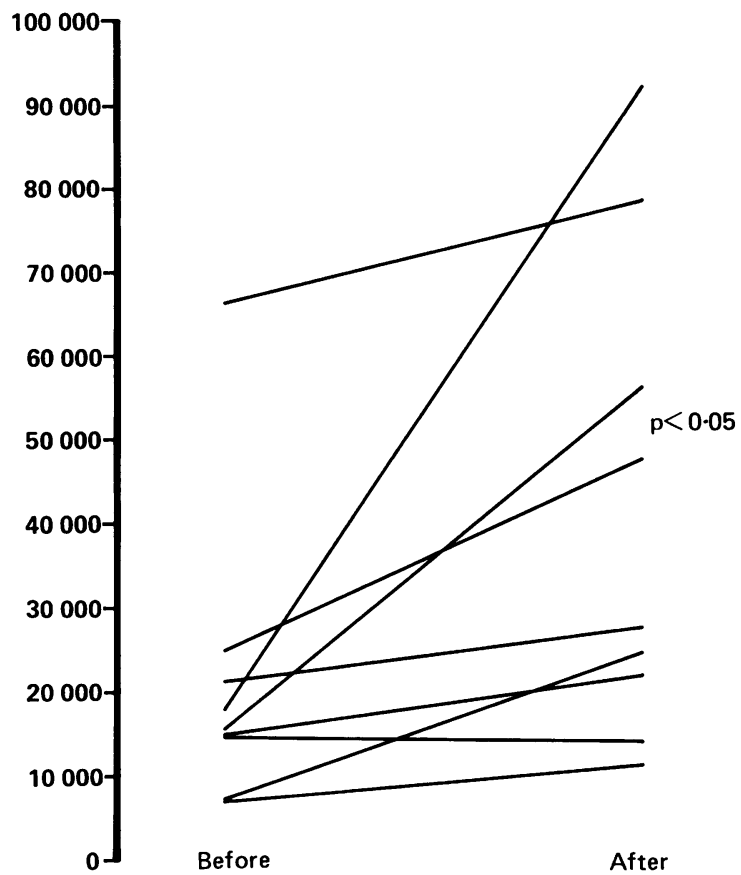

Fig. 6 Change in quantitative SVA energy values after cisapride in the fasting phase. The graph shows the relative change in nine subjects, of fasting quantitative SVA energy values before and after cisapride. $p<0.05$ by Mann Whitney Utest.

greater sensitivity to transmitted energy within a frequency range extending beyond the acoustic, and low susceptibility to any airborne background noise.

Initially, the method was designed to capture energy within a spectral range of $2-60000 \mathrm{~Hz}$, because there was little published work to indicate the optimum. The initial dominant signal which was found, however, had the rapid rate of rise and decay consistent with an energy pulse, was synchronous with the directly monitored heartbeat and was considered to represent a vascular pulsation, most likely emanating from the aorta (Fig. 1). Fortunately, this high energy signal, which was unwanted in these studies, had a low frequency range and it was possible to exclude it by the incorporation of a low frequency four pole high pass filter. In addition, the wide bandwidth initially utilised was accompanied by some high frequency electronic noise, and therefore, some high frequency attenuation was also incorporated, giving the system a flat response between $40-10000 \mathrm{~Hz}$.

With a maximum signal frequency of $10 \mathrm{KHz}$, it became clear that accurate processing and measurement of lengthy signals by conventional analogue to digital conversion would require manipulation of vast 
data quantities. Indeed, previous work which has addressed the concept of digitisation and computerisation of bowel sound energy was limited by these technical considerations, to analysis of very short duration signals - for example, 5-20 milliseconds duration $^{910}$ which rendered meaningful interpretation impossible.

We therefore, chose to approach this problem by a method involving total data integration over set intervals of five seconds, which was then addressed to the inbuilt analogue to digital converter of a BBC microcomputer (sampling rate $100 \mathrm{~Hz}$ ). This method allowed accurate digitisation of the integrated signal and permitted capture and analysis of long duration signals for evaluation against conventional tests of gastrointestinal activity. Indeed, this study has for the first time shown a correlation between vibrational energy which can be captured and measured at the abdominal surface and oral to caecal transit times for solids. These findings suggest a relationship between vibrational energy detected by this method, and propulsive gastrointestinal motor function.

While this observation is important, further exposition must at this point, be speculative. The SVA system addresses fluctuating mechanical pressure waves, at the abdominal surface, emanating from intra-abdominal sources and the magnitude of these changes is related to both the force and motion, associated with the signal source. ${ }^{1}$ Thus from this principle, gastrointestinal contractions associated with propulsion or retropulsion of intraluminal contents would be expected to yield a greater signal than segmental contractions. Furthermore, the observations in this study have largely concerned the fed phase of gastrointestinal activity, in which propulsive contractions are known to predominate. ${ }^{14}$ Thus, if our hypothesis that the SVA system preferentially addresses propulsive activity were correct, then it could explain the observation of this study of a significant correlation between quantitative SVA values and oral to caecal transit time of solids.

The system's graphic display allows qualitative assessment of captured signals. In the postprandial state, a variable high energy peak was seen immediately after the pause for meal ingestion, which lasted 20-60 minutes, with a more gradual return to fasting activity thereafter (Fig. 5). These findings bear some resemblance to those of Gill et al ${ }^{15}$ who found by intraluminal manometric studies, that the duration of postprandial motor activity in the gastric antrum and duodenum varied from 50 (13) minutes after small solid meals to 147 (38) minutes after larger solid meals and was maximal in intensity shortly after the meal had been ingested.

Measurement of drug effects on gastrointestinal motor function, is conventionally, very difficult.
Because our studies had shown a correlation between quantitative SVA measurements and propulsive activity (transit times), it was decided to proceed with an investigation of the system's sensitivity for detection of changes induced by a known prokinetic gastrointestinal stimulant (cisapride). In the event, cisapride produced a significant quantitative SVA response in the fasting phase in the 'gas bloat' patients. In the fed state, cisapride promoted GI activity, with a significant acceleration of intestinal transit, but a non-significant increase in postprandial SVA values. This latter discrepancy may be related to the small number of subjects concerned. It is clear, from the initial solid meal study that although a significant correlation exists between SVA values and transit, the correlation coefficient does not approach unity. Thus, the relationship may not be evident when patient numbers are small, as in the present drug stimulation study. The findings are nonetheless encouraging, and it is possible that with development, that this system could prove useful for evaluation of drug effects on the gastrointestinal tract in a clinical or experimental context.

In conclusion, the findings of these studies provide strong evidence that the energy which is captured and measured by the SVA system, emanates from the gastrointestinal tract and is related to propagative activity. These findings raise certain questions, however, concerning the physics of high frequency signal generation in the gut, the proportional organ contribution to the total signal, and the relationship, if any, of SVA signals in the fasting phase to the migrating motor complex. We hope to address some or all of these questions in subsequent studies.

The authors gratefully acknowledge the technical advice of Dr Nigel Orr, Department of Clinical Physics and Bioengineering, University of Glasgow and Professor R A B Mollan, Department of Orthopaedic Surgery, Queen's University, Belfast.

\section{References}

1 Bishop RED. Vibration. Cambridge: Cambridge University Press, Cambridge 1979: 1-20.

2 Cannon WB. Auscultation of the rhythmic sounds produced by the stomach and intestines. Am J Physiol 1905; 14: 339-53.

$3 \mathrm{Du}$ Plessis DJ. Clinical observations on intestinal motility. S Afr Med J 1954; 28: 27-33.

4 Baker LW, Dudley HAF. Auscultation of the abdomen in surgical patients. Lancet 1961; ii: 517-9.

5 Horn GE, Mynors JM. Recording the bowel sounds. Med Biol Eng 1966; 4: 205-8.

6 Wells C, Rawlinson K, Tinckler L, Jones H, Saunders J. Ileus and post-operative intestinal motility. Lancet 1961; ii: $136-7$. 
7 Farrar JT, Ingelfinger FJ. Gastrointestinal motility as revealed by study of abdominal sounds. Gastroenterology 1955; 29: 789-900.

8 Watson WC, Knox Elizabeth C. Phonenterography: the recording and analysis of bowel sounds. Gut 1967; 8: 8894.

9 Politzer JP, Devroede E, Vasseur C, Gerard J, Thibault R. The genesis of bowel sounds: influence of viscus and gastrointestinal content. Gastroenterology 1976; 71: $282-5$.

10 Dalle D, Devroede G, Thibault R, et al. Computer analysis of bowel sounds. Comput Biol Med 1975; 4: 247-56.
11 Brigham EO. The fast fourier transform. New York: Prentice-Hall, 1974.

12 Bond JH, Levitt MD, Prentiss R. Investigation of small bowel transit time in man utilizing pulmonary hydrogen (H2) measurements. J Lab Clin Med 1975; 85: 546-55.

13 Campbell FC, Blanch Geraldine, Cook D, Cuschieri A. Critical rise in breath hydrogen in evaluation of intestinal transit [Abstract]. Gut 1986; 10: A1270.

14 Summers RW, Dusdeiker NS. Computer generated display of longtitudonal spike burst spread in the small intestine. In: Christensen $J$, ed. Gastrointestinal motility. New York: Raven Press, 1980: 339-44.

15 Gill RC, Kellow JE, Wingate DL. The migrating motor complex at home. Gastroenterology 1987; 92: 1405. 